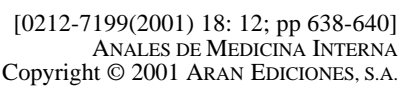

Copyright () 2001 ARAN EDICIONES, S.A.

An. Med. INTERNa (Madrid) Vol. 18, N. ${ }^{\circ} 12$, pp. 638-640, 2001

\title{
Fibrosis mediastínica idiopática asociada a hipercoagulabilidad. A propósito de un caso
}

\author{
A. BAUZÁ ALONSO, J. P. GARCÍA GÓMEZ, C. ABADES VÁZQUEZ, N. GARCÍA \\ GONZÁLEZ, J. PRIETO VALTUEÑA
}

Departamento de Medicina Interna. Clínica Universitaria de Navarra. Facultad de Medicina. Universidad de Navarra.

\author{
IDIOPATIC MEDIASTINAL FIBROSIS IN ASSOCIATION WITH \\ HYPERCOAGULABILITY STATE. A CASE REPORT
}

\begin{abstract}
RESUMEN
La fibrosis mediastínica es una entidad de baja frecuencia y de etiología desconocida en la mayor parte de los casos. Se caracteriza por la presencia de una masa fibrótica en el mediastino que puede invadir estructuras vecinas tales como el esófago, la tráquea, el nervio recurrente, la vena cava superior etc. Solamente un 1-2\% de pacientes con fibrosis mediastínica presentan un cuadro de obstrucción de vena cava superior, y esta es producida en casi todos los casos por compresión extrínseca por el magma fibrótico.

Se presenta el caso de un paciente con un trastorno de hipercoagulabilidad asociado a fibrosis mediastínica con obstrucción de la vena cava superior por trombosis (mecanismo intrínseco) así como su evolución satisfactoria tras el tratamiento combinado con anticoagulación oral y tamoxifeno.
\end{abstract}

PALABRAS CLAVE: Fibrosis mediatínica. Hipercoagulabilidad. Tamoxifeno.

\begin{abstract}
Mediastinal fibrosis is a rare and unknown disease characterized by the presence of a fibrotic mass in the anterior mediastinum that can inva de close structures as gullet, trachea, recurrent nerve, superior cava vein etc. Only 1-2\% of patients with mediastinal fibrosis associate superior cava vein obstruction. When it appears, it is produced by extrinsic com pression for fibrotic magma in almost all the cases. A case of hypercoa gulability disorder associated with mediastinal fibrosis and superior cava vein obstruction by thrombosis (intrinsec mechanism), and the satisfactory evolution after the treatment with oral anticoagulation, cor ticosteroids and tamoxifen is presented.
\end{abstract}

KEY WORDS:Mediastinal fibrosis. Hypercoagulability. Tamoxifen.

Bauzá Alonso A, García Gómez JP, Abades Vázquez C, García González N, Prieto Valtueña J. Fibrosis mediastínica idiopática asociada a hipercoagulabilidad. A propósito de un caso. An Med Interna (Madrid) 2001; 18: 638-640.

\section{INTRODUCCIÓN}

La fibrosis mediastínica es una enfermedad rara y benigna (1) que se incluye dentro del grupo de las fibrosis idiopáticas sistémicas (2). Es el estadio final de un cuadro inicialmente inflamatorio (hallazgo histológico de linfocitos y PMN) (3) que evoluciona hacia una esclerosis del mediastino, con depósito de una sustancia colágena densa (4) que produce compresión de estructuras vecinas tales como la vía aérea, el nervio laríngeo recurrente, el nervio frénico, el esófago, la arteria pulmonar, las venas pulmonares próximas o la vena cava superior (1-2\% de los pacientes) (5).

La mayoría de los casos se han atribuído clásicamente a tuberculosis o histoplasmosis, así como a sarcoidosis, silicosis, lúes, actinomicosis (6) y otras enfermedades fúngicas. Actualmente se consideran otras causas, tales como aneurismas aórticos, traumatismos, lupus eritematoso sistémico, $\mathrm{S}$. de Sjögren, tumores o fármacos (metisergida $(2,4)$, alfa-metildopa, hidralacina, bromocriptina, $\beta$-bloqueantes o antineoplásicos (2)). En la mayor parte de los casos la etiología es desconocida (7). Raras veces es posible hacer un diagnóstico etiológico preciso, pues una infección previa puede preceder al proceso crónico en meses o años (8). Puede asociarse a fibrosis en otras localizaciones (retroperitoneal, mesentérica, cervicofacial) o a otros procesos fibrosantes como enfermedad de Peyronie o enfermedad de Dupuytren (2).

La patogenia no es bien conocida. Algunos autores han sugerido que la fibrosis mediastínica es consecuencia de una reacción de hipersensibilidad, en la que un antígeno estimularía la actividad fibroblástica alrededor de los granulomas. Otros han opinado que la fibrosis mediastínica surgiría por la ruptura traumática de adenopatías granulomatosas, con el vertido de sus contenidos irritantes en tejidos blandos adyacentes (6).

Trabajo aceptado: 12 de Mayo de 2000

Correspondencia: Ana Bauzá Alonso. Pío XII s/n 31008. Pamplona. Telf: 948- 255400. Fax: 948296500, Correo electrónico: anabauza@unav.es 
Los pacientes, cuya edad suele estar entre los 30-60 años, presentan una clínica inespecífica con astenia, anorexia, febrícula, tos irritativa y dolor torácico como síntomas más frecuentes. Sin embargo, hasta el $40 \%$ de los pacientes están asintomáticos (3), sugiriendo el diagnóstico una VSG elevada, anemia moderada y la presencia de una masa mediastínica objetivada por técnicas de imagen (TAC, RM). El diagnóstico definitivo es anátomo-patológico (4).

El tratamiento quirúrgico en estos pacientes está muy limitado, especialmente cuando presentan obstrucción vascular avanzada. En la mayor parte de los casos el tratamiento es paliativo de las complicaciones (6). Se ha utilizado tratamiento médico con corticoides, a los que pueden añadirse ciclofosfamida o azatriopina si la respuesta no es satisfactoria (2)

\section{CASO APORTADO}

Paciente varón de 25 años de edad que presentó un episodio de tromboflebitis en extremidad inferior derecha hace cuatro años. Un mes después presentó un episodio de trombosis de seno longitudinal craneal, diagnosticado por RM cerebral, que se manifestó por un cuadro de hipertensión intracraneal (cefalea intensa, pérdida de campo visual y desviación del ojo izquierdo en aducción). Fue tratado mediante anticoagulación e implantación de válvula lumboperitoneal.

El paciente se encontró asintomático hasta hace dieciocho meses, en que presentó un nuevo episodio de tromboflebitis en extremidad inferior izquierda. Tres meses después, cuadro catarral con tos irritativa y febrícula vespertina, prácticamente diaria, que en ocasiones llegó a ser fiebre de hasta $39^{\circ} \mathrm{C}$. Se acompañó de escalofríos, intensa astenia, mialgias cervicales y lumbares, y pérdida de $4 \mathrm{~kg}$ de peso. En el último mes apareció edema facial que aumentaba con el decúbito nocturno.

La exploración física mostró palidez cutáneo-mucosa y edema facial importante, así como múltiples aftas no dolorosas en bordes laterales de la lengua y una microadenopatía axilar derecha. La auscultación cardíaca presentó taquicardia y la auscultación pulmonar ligera hipoventilación en base derecha. El resto de la exploración no mostró alteraciones.

El estudio realizado al paciente mostró los siguientes resultados: anemia microcítica e hipocrómica; ligera leucocitosis con netrofilia (74,3\%); elevada VSG (126/133); proteinograma con hipoalbuminemia y aumento de alfa 2 , beta y gamma globulinas; ferropenia con ferritina elevada y capacidad de fijación disminuída; resto de bioquímica normal. El estudio de coagulación (incluyendo análisis molecular de los factores $\mathrm{V}$ y protrombina) fué normal. Las serologías de hepatitis (B, C, A), VIH, salmonella, brucella, toxoplasma, CMV, VEB fueron negativas.

ANA positivos a título 1/80. ANCA, RNP, RO, LA, anti-DNA, anti-sm, FR, crioglobulinas, anti-cardiolipina, anti-coagulante lúpico, Coombs directo e indirecto negativos.

HLA-AB: A2, A26, B45, B72, BW6.

Se realizó TAC cervico-torácico que mostró la presencia de adenopatías cervicales y paratraqueales, derrame pleural derecho, y una masa mediastínica (Fig. 1), así como trombosis de vena cava superior (Fig. 2) y yugular interna derecha. En el TAC abdominal no se observaron adenopatías. En el estudio digestivo (colonoscopia y gastroscopia) aparecieron varices esofágicas III/IV en tercio superior y medio. Las biopsias de ileon y duodeno fueron normales al igual que el ecocardiograma y ECG.

Se practicó mediastinoscopia con biopsia de la masa mediastínica, con el diagnóstico anátomo-patológico de fibrosis con inflamación crónica y presencia de células gigantes de cuerpo extraño.

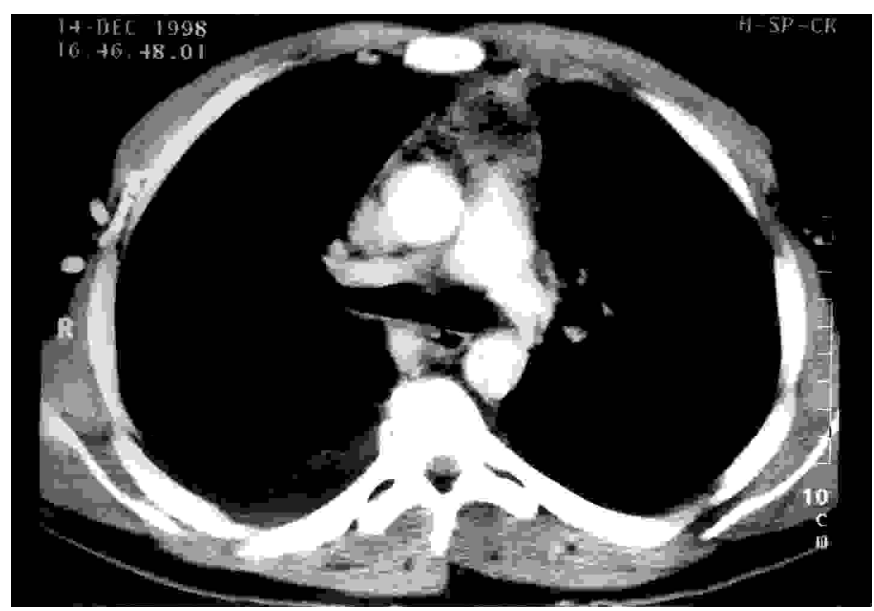

Fig. 1. Masa que ocupa mediastino anterior, con presencia de necrosis y burbujas en su interior en relación con mediastinoscopia previa. En la parte inferior se aprecia atelectasia pasiva secundaria a derrame pleural derecho.

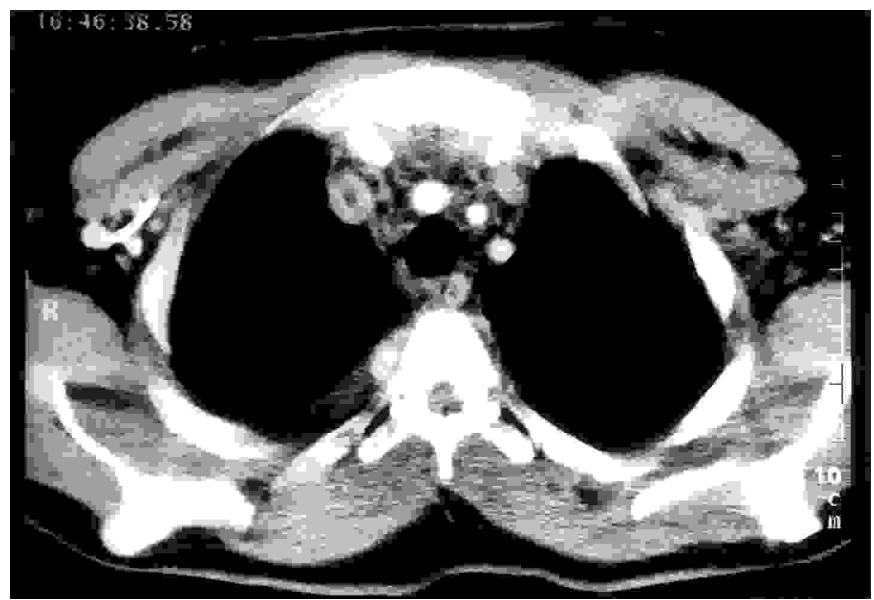

Fig. 2. Trombosis de vena cava superior.

\section{DISCUSIÓN}

El caso que se presenta corresponde a un varón joven, cuyo cuadro clínico se caracteriza por la asociación excepcional de fibrosis mediastínica e hipercoagulabilidad de causa desconocida. Dicho estado de hipercoagulabilidad, de varios años de evolución, se manifestó por repetidos episodios de trombosis a distintos niveles: tromboflebitis en extremidad inferior derecha, trombosis de seno longitudinal superior y trombosis de vena cava superior. El paciente desarrolló en pocos meses una obstrucción de la vena cava superior por trombosis, con aparición de varices esofágicas (III/IV) en tercio superior del esófago, sin que los medios de imagen hayan evidenciado invasión de la vena cava u otras estructuras por la masa fibrótica.

La obstrucción de la vena cava superior es en sí misma un síndrome infrecuente y su diagnóstico clínico suele ser tardío. Su causa más frecuente son las neoplasias, sobre todo carcinoma de pulmón y linfomas. Menos frecuentemente se asocia a patología benigna como la fibrosis mediastínica (9). Sólo 
el 1-2\% de pacientes con fibrosis mediastínica presentan además obstrucción de la vena cava superior producida por la compresión extrínseca del magma fibrótico sobre la vena cava.

Otras enfermedades pueden producir obstrucción de la vena cava por trombosis (obstrucción intrínseca). En una de las series más amplias publicada (10), la causa más frecuente fue la enfermedad de Behçet (64\%), encontrándose con menor frecuencia carcinoma de pulmón (14\%), tuberculosis mediastínica (14\%), o LES $(3,5 \%)$. Hasta en un $7 \%$ de los casos la etiología no pudo determinarse.

La enfermedad de Behçet asocia frecuentemente tromboflebitis superficiales y con menor frecuencia trombosis del sistema venoso profundo tales como vena cava superior, inferior u otras. Ha sido descrito algún caso de asociación de enfermedad de Behçet con fibrosis mediastínica y obstrucción de vena cava superior (11). El caso que se presenta no cumplía ninguno de los criterios diagnósticos de enfermedad de Behçet. Por otra parte, todos los estudios realizados para etiquetar la causa de la hipercoagulabilidad, incluyendo el estudio molecular del factor $\mathrm{V} y$ protrombina fueron negativos.
Se describe por consiguiente una rara asociación de fibrosis mediastínica e hipercoagulabilidad de etiología desconocida.

Se decidió realizar tratamiento de prueba con corticoides, anticoagulación oral y tamoxifeno, siendo este último un fármaco con acción antifibrogénica que ha sido ensayado en otras enfermedades fibrosantes como la fibrosis retroperitoneal $(12,13)$. Tras 4 meses de tratamiento el paciente ha presentado una importante mejoría de su estado general, con ganancia ponderal y desaparición de los accesos febriles que presentaba anteriormente. Se ha practicado analítica de control con recuperación de los niveles de hemoglobina y una disminución muy evidente de la VSG. El TAC torácico ha puesto de manifiesto una reducción del volumen de la masa del mediastino superior al $50 \%$, con ausencia de derrame pleural y persistencia de la trombosis de vena cava superior.

Esta respuesta al tratamiento combinado con corticoides y tamoxifeno sugiere su utilidad como un arma terapéutica más en el tratamiento de la fibrosis mediastínica y hace necesarios estudios más amplios para confirmar su eficacia.

\section{Bibliografía}

1. Mitchel-IM, Saunders-NR, Mather-O, Lennox-SC, Walker-DR. Surgical treatment of idiopatic mediastinal fibrosis. Report of five cases. Thorax 1986; 41: 210-4.

2. López Soto A. Fibrosis idiopáticas sistémicas. En: Farreras-Rozman. Medicina Interna. Mosby/Doyma, Madrid. 1995; 13 a ed. 1123.

3. Wold LE, Weiland LH. Mediastinitis crónica y fibrosis mediastínica idiopática Am J Surg Pathol 1983; 7: 477-82.

4. Batten J. Mediastinitis crónica y fibrosis mediastínica idiopática. En: Price's. Medicina Interna. Ed. Espaxs S.A. Barcelona 1982; 1246-7.

5. Peters P, Saborowski F, Seel R, Arnold G, Herse B. Aggressive mediastinal fibrosis, a rare cause of superior vena cava obstruction. Case report and review of the literature. Z Kardiol 1988; 77: 194-7

6. Roca Calvo MJ. Fibrosis mediastínica. Rev Clin Esp 1993; 192: 327-8.

7. Fosse E, Fjeld NB, Arnkvaern R, Semb G, Sauer T. Mediastinal fibrosis. A case report. J Oslo City Hosp 1989; 39: 103-6.

8. Ian RG. Dowdeswell. Mediastinitis crónica. En: Jay H. Stein. Medicina Interna. $3^{a}$ ed. Salvat editores; Barcelona 1991; Cap. 95: 749.

9. Fassin D, Wechsler B, Cabane J, Godeau P. Superior caval syndrome. Diagnostic and therapeutic management. A propos of 20 cases. RevMed Interna 1985; 6: 27-35.

10. Ousehal A, Essadki O, Abdelouafi A, Kadiri R. Superior vena cava thrombosis. Radiological aspects. A proposit of 28 cases. Am Radiol Paris 1993; 36: 303-9

11. Abid R, Hadj Taieb Abid F, Chaabane M, Boussen K, Robbana A, Bouhaouala $\mathrm{H}$, et al. Superior caval syndrome caused by cronic mediastinitis in Behçet's disease. J Radiol 1995; 76: 155-7.

12. Owens LV, Cance WG, Huth JF. Retroperitoneal fibrosis treated with tamoxifen. Am-Surg 1995; 61: 842-4.

13. Tonietto-G, Agresta F, Della Libera D, Bittesini L. Treatment of idiopathic retroperitoneal fibrosis by tamoxifen. Eur J Surg 1997; 163: 231-5. 